BARROSO, R.M.V. et al. Análise morfométrica do forame mandibular em Cachorro do Mato Vinagre (Speothos venaticus, LUND 1842). PUBVET, Londrina, V. 8, N. 14, Ed. 263, Art. 1744, Julho, 2014.

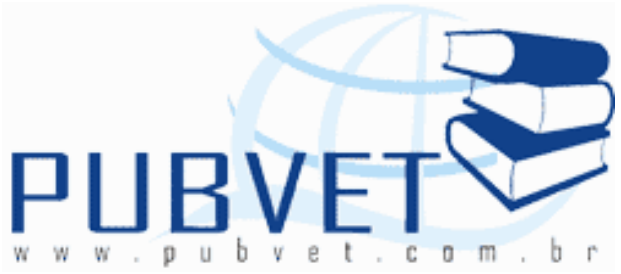

PUBVET, Publicações em Medicina Veterinária e Zootecnia.

\title{
Análise morfométrica do forame mandibular em Cachorro do Mato Vinagre (Speothos venaticus, LUND 1842)
}

\author{
Rogério Magno do Vale Barroso ${ }^{1}$, André Luis Quagliatto Santos ${ }^{2}$, Rodrigo \\ Kruger $^{3}$,Gustavo Pedro Milanezzi ${ }^{4}$, Juliano Saich Kurtt ${ }^{4}$, Ednaldo Carvalho \\ Guimarães $^{5}$
}

${ }^{1}$ Professor Mestre, Coordenador do Curso de Medicina Veterinária da Escola Superior São Francisco de Assis - ESFA, Doutorando em Ciências Veterinárias. UFU. vetbarroso@yahoo.com.br

${ }^{2}$ Professor Doutor, Coordenador do Laboratório de Pesquisa em Animais Silvestres - LAPAS e orientador do programa de pós graduação em Ciências Veterinárias - UFU

${ }^{3}$ Aluno de graduação em Medicina Veterinária da Escola Superior São Francisco de Assis - ESFA, bolsista de iniciação científica Fundação de Amparo a Pesquisa do Espírito Santo - FAPES

${ }^{4}$ Alunos de graduação em Medicina Veterinária da Escola Superior São Francisco de Assis - ESFA, voluntários do programa de iniciação científica ${ }^{5}$ Professor Doutor,Faculdade de Matemática - Universidade Federal de Uberlândia

\section{Resumo}

Objetivou-se, avaliar morfometricamente o forame mandibular, em espécimes de Cachorro do Mato Vinagre (Speothos Venaticus) visando compreender de 
BARROSO, R.M.V. et al. Análise morfométrica do forame mandibular em Cachorro do Mato Vinagre (Speothos venaticus, LUND 1842). PUBVET, Londrina, V. 8, N. 14, Ed. 263, Art. 1744, Julho, 2014.

forma mais precisa sua localização, para que tenhamos um bloqueio anestésico local mais efetivo do nervo alveolar mandibular. Foram utilizados 20 hemimandíbulas de Cachorro do Mato Vinagre (Speothos Venaticus) adultos, sem definição de sexo. Com auxílio de um paquímetro milimetrado tipo universal, foram mensurados 6 pontos de referência, LONG (da borda mais lateral do processo condilar até o primeiro dente incisivo inferior); TRANS (da borda ventral até a borda dorsal da mandíbula entre o segundo dente pré-molar e o primeiro dente molar inferiores); FVENTRAL (da borda mais rostral do forame mandibular até a borda ventral da mandíbula); ANG (da extremidade da borda mais rostral do forame mandibular até o processo angular da mandíbula); COND (extremidade da borda mais rostral do forame mandibular até a borda medial do processo condilar da mandíbula) e COR (da borda mais rostral do forame mandibular até a borda medial do processo condilar da mandíbula). Os dados obtidos foram analisados através de estatística descritiva e, também pelo o teste $T$ de Student com $p=0,05$ onde observou-se que para um melhor bloqueio anestésico local do nervo alveolar inferior a agulha deverá ser inserida de 21,45 a $21,94 \mathrm{~mm}$ a partir do processo angular e 13,11 a 14,01 $\mathrm{mm}$ da borda ventral da mandíbula.

Palavras-chave: Speothos Venaticus, forame mandibular, morfometria, analgesia.

\title{
Morphometric analysis of the mandibular foramen of Bush Dog (Speothos venaticus, LUND 1842)
}

\begin{abstract}
The objective of this work was to perform a morphometric evaluation of the mandibular foramen of Bush Dog in order to establish its more precise topography and to offer subsides for a more effective local anesthetic blockage of the mandibular alveolar nerve. Twenty hemimandibles of Bush Dog (Speothos Venaticus) were used. With the aid of a universal caliper rule, the 6
\end{abstract}


BARROSO, R.M.V. et al. Análise morfométrica do forame mandibular em Cachorro do Mato Vinagre (Speothos venaticus, LUND 1842). PUBVET, Londrina, V. 8, N. 14, Ed. 263, Art. 1744, Julho, 2014.

reference points were measured: LONG (from the most lateral margin of the condylar process up to the first inferior incisor tooth); TRANS (from the ventral margin up to the dorsal mandible margin between the second premolar tooth and the first inferior molar tooth); FVENTRAL (from the most rostral margin of the mandibular foramen up to the ventral mandibular margin); ANG (from the end of the most rostral margin of the mandibular foramen up to the mandible angular process), COND (from the end of the most rostral margin of the mandibular foramen up to the medial margin of the mandible condylar process) and COR (from the most rostral margin of the mandibular foramen up to the medial margin of the mandible cononoid process). Following, the data obtained were submitted to descriptive statistical analysis and to the student's t-test with $p=0.05$. It was observed that for a better local anesthetic blockage of the inferior alveolar nerve, the needle should be introduced 13,11 to 14,01 $\mathrm{mm}$ from the mandible ventral margin and 21,45 to $21,94 \mathrm{~mm}$ from the angular process.

Keywords: Speothos Venaticus, forame mandibular, morfometria, analgesia.

\section{INTRODUÇÃO}

Distribuindo-se do Panamá até o sul do Brasil e norte da Argentina, o Cachorro do Mato Vinagre é o único representante atual do gênero Speothos (BEISIEGEL \& ZUERCHER, 2005).

Speothos venaticus é considerada naturalmente rara ao longo de toda sua distribuição (DEMATTEO \& LOISELLE 2008) e atualmente é considerada próxima de ameaçada (Near threatened - NT) pela IUCN (ZUERCHER et al. 2008). No Brasil, o cachorro-vinagre é considerado Vulnerável à extinção (VU), e como principais ameaças são citadas a alteração e fragmentação do habitat e a caça de suas presas naturais (OLIVEIRA \& DALPONTE 2008). A transmissão de doenças por canídeos domésticos também é apontada como uma potencial ameaça nas áreas onde as espécies entram em contato (OLIVEIRA \& DALPONTE 2008; OLIVEIRA 2009). 
BARROSO, R.M.V. et al. Análise morfométrica do forame mandibular em Cachorro do Mato Vinagre (Speothos venaticus, LUND 1842). PUBVET, Londrina, V. 8, N. 14, Ed. 263, Art. 1744, Julho, 2014.

O cachorro-vinagre possui diversas diferenças morfológicas em relação aos outros canídeos, como a presença de membranas interdigitais, supostamente como adaptação à natação e para o caminhar em solos moles próximos a cursos d'água (BEISIEGEL \& ZUERCHER 2005). O comportamento da espécie é também diferenciado do de outros canídeos neotropicais, pois esta espécie apresenta a formação de grupos familiares contendo entre dois e doze indivíduos, com a presença de um único casal reprodutivo monogâmico por grupo (BEISIEGEL \& ZUERCHER 2005; KLEIMAN 1972).

Há muito pouco conhecimento sobre a espécie em ambiente natural, o que dificulta a proposição de medidas de conservação e o correto acesso a seu status de ameaça (DEMATTEO \& LOISELLE 2008). Sabe-se que atualmente a maior parte das populações naturais do cachorro-vinagre encontra-se em áreas fora de Unidades de Conservação, ao longo de toda sua distribuição (DEMATTEO \& LOISELLE 2008).

Segundo Souza Júnior, et al. (2013), uma das causas mais comuns de atendimentos veterinários em carnívoros silvestres destina-se ao tratamento de afecções dentárias. Os traumas com exposição da polpa dentária e conseqüente fístula mandibular são muito freqüentes e podem decorrer de acidentes em grades e concreto dos recintos, brigas com outros animais ou ainda por efeito de ação deletéria de humanos (corte de dentes). Procedimentos que visam preservar a dentição e a oclusão mantêm a integridade do aparelho mastigatório e asseguram a ingestão adequada de alimentos.

A localização do forame mandibular em carnívoros se dá na face medial do ramo da mandíbula e ventralmente ao ponto de inserção do músculo temporal. O forame mandibular dá origem a um canal que se encerra nos forames mentonianos, permitindo a passagem da artéria, veia e nervo alveolares inferiores (GETTY, 1986).

O nervo alveolar inferior é um nervo sensitivo, ramo do nervo mandibular, que após sua origem segue ventralmente por uma distância de 
BARROSO, R.M.V. et al. Análise morfométrica do forame mandibular em Cachorro do Mato Vinagre (Speothos venaticus, LUND 1842). PUBVET, Londrina, V. 8, N. 14, Ed. 263, Art. 1744, Julho, 2014.

aproximadamente 10 milímetros, antes de penetrar no forame mandibular (GETTY, 1986).

Dentro do canal mandibular o nervo alveolar inferior divide-se em ramos caudais, médios e rostrais, sendo que estes ramos inervam os dentes molares, pré-molares, caninos e incisivos e as gengivas correspondentes (GETTY, 1986).

O bloqueio anestésico realizado no nervo alveolar inferior promove anestesia nos dentes pré-molares, caninos, incisivos, pele e mucosa da bochecha e lábio inferior (MUIR et al., 2001).

Conhecer a localização do forame mandibular é essencial para o bloqueio anestésico local dos nervos mandibular e alveolar inferior (NICHOLSON, 1985; NICKEL et al., 1981; BLANTON \& RODA, 1995; MILKEN, 2003).

A aplicação de anestésicos locais antes da produção do trauma cirúrgico limita ou minimiza a reação tissular inflamatória e diminui a sensibilidade central à dor, proporcionando ao paciente maior conforto pós-operatório (MILKEN, et al, 2003).

Diversos autores divergem sobre a exata localização do forame mandibular em pequenos animais, e somente Gross et al. (2000) especificou que o estudo foi realizado em gatos. Skarda (1996) e Fantoni \& Cortopassi (2002) indicam a posição a $5 \mathrm{~mm}$ rostral ao processo angular e de 10 a $20 \mathrm{~mm}$ dorsal a margem da mandíbula. Já Muir et al. (2001) afirma que o posicionamento se dá a $15 \mathrm{~mm}$ rostralmente ao processo angular e $15 \mathrm{~mm}$ dorsalmente a margem da mandíbula.

O objetivo deste trabalho é avaliar morfometricamente o forame mandibular, em Cachorro do Mato Vinagre (Speothos Venaticus), visando ainda estabelecer de forma mais precisa sua localização, e oferecer subsídios para um bloqueio anestésico local mais efetivo do nervo alveolar mandibular. 
BARROSO, R.M.V. et al. Análise morfométrica do forame mandibular em Cachorro do Mato Vinagre (Speothos venaticus, LUND 1842). PUBVET, Londrina, V. 8, N. 14, Ed. 263, Art. 1744, Julho, 2014.

\section{MATERIAL E MÉTODOS}

Foram utilizadas 20 hemi-mandíbulas (10 mandíbulas), de Cachorro do Mato Vinagre provenientes do acervo ósseo do Museu Melo Leitão e também do laboratório de anatomia animal da Escola Superior São Francisco de Assis. Estas mandíbulas foram coletadas ao longo dos anos e estão catalogadas nas referidas Instituições.

Com auxílio de um paquímetro milimetrado tipo universal, (Vernier Caliper, marca Marberg, foram mensurados 6 pontos de referência (FIGURA 1), adotando a abreviaturas LONG (medida realizada da margem mais lateral do processo condilar até o primeiro dente incisivo inferior); TRANS (medida realizada da margem ventral da mandíbula até a margem dorsal entre o quarto dente pré-molar e o primeiro dente molar inferior); FVENTRAL (medida realizada da margem rostral do forame mandibular até a margem ventral da mandíbula); ANG (medida realizada da extremidade da margem mais rostral do forame mandibular até o processo angular da mandíbula), COND (medida realizada da extremidade da margem mais rostral do forame mandibular até a margem medial do processo condilar da mandíbula) e COR (medida realizada da margem mais rostral do forame mandibular até a margem medial do processo coronóide da mandíbula). Os dados obtidos foram submetido à análise estatística descritiva e ainda aplicou-se $\mathrm{o}$ teste $\mathrm{t}$ de Student com $p=0,05$. 
BARROSO, R.M.V. et al. Análise morfométrica do forame mandibular em Cachorro do Mato Vinagre (Speothos venaticus, LUND 1842). PUBVET, Londrina, V. 8, N. 14, Ed. 263, Art. 1744, Julho, 2014.

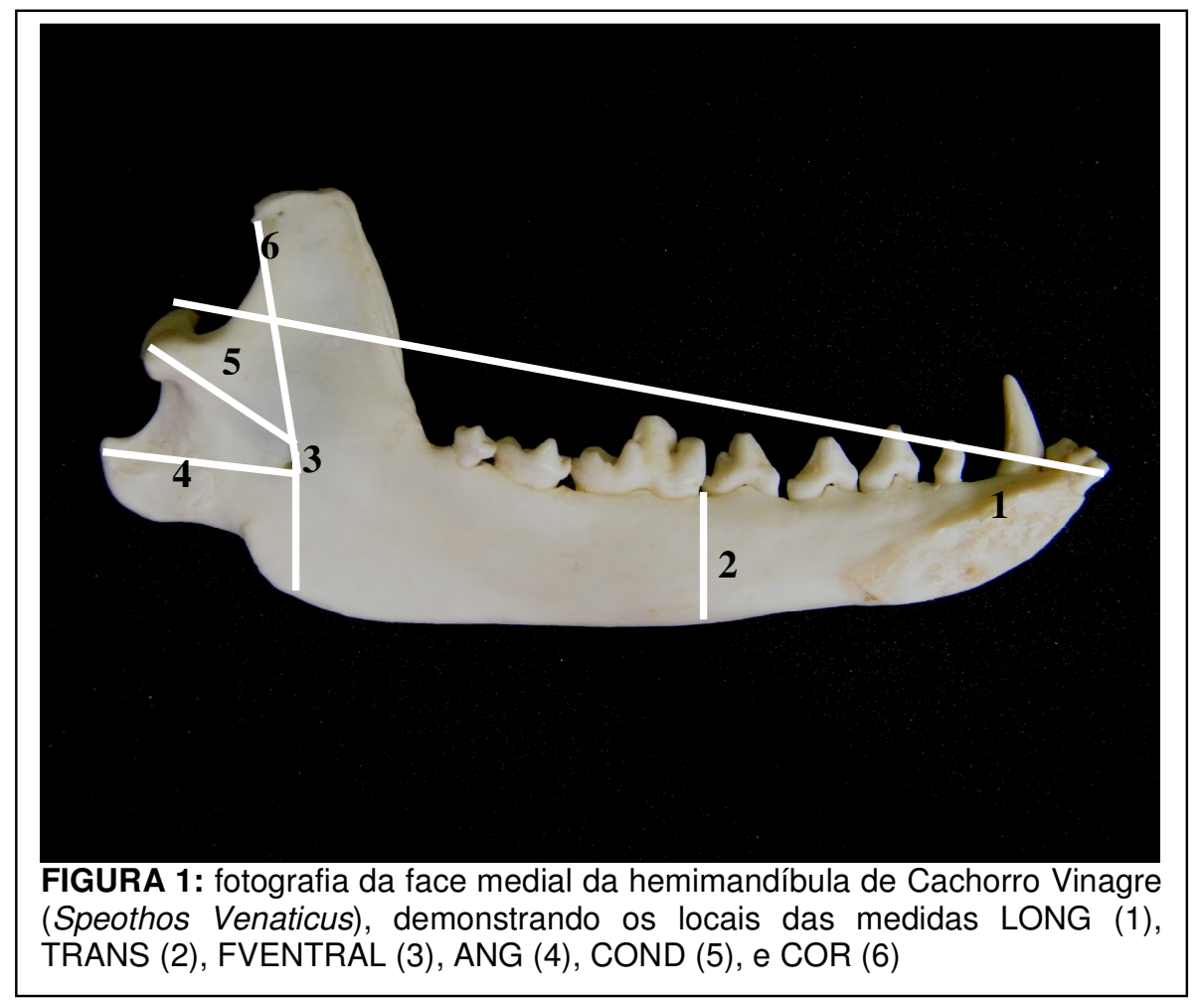

\section{RESULTADOS}

Conforme demonstrado na Tabela I, considerando análise estatística descritiva e pelo teste t-Student com $\mathrm{p}=0,05$ e intervalo de confiança de 95\%, encontramos para a variável LONG direita uma média de 100,12mm com desvio padrão de 4,01 mm e para LONG esquerda uma média de 101,14 mm com desvio padrão de 4,24 mm e na comparação entre os dois antímeros, $p=0,3201$, não havendo, portanto diferença significativa. 
BARROSO, R.M.V. et al. Análise morfométrica do forame mandibular em Cachorro do Mato Vinagre (Speothos venaticus, LUND 1842). PUBVET, Londrina, V. 8, N. 14, Ed. 263, Art. 1744, Julho, 2014.

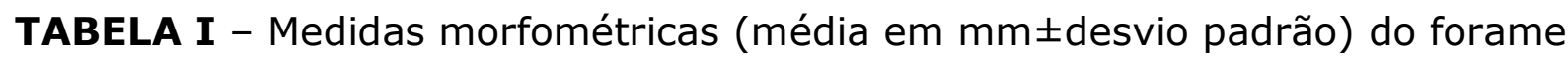
mandibular de Cachorro do Mato Vinagre (Speothos Venaticus) hemimandíbulas direita e esquerda e valor de $p$ obtido através do teste $t$ de student.

\begin{tabular}{lccc}
\hline Ponto Mensurado & $\begin{array}{c}\text { Hemimandíbula Direita } \\
\text { Média } \pm \text { Desvio Padrão }\end{array}$ & $\begin{array}{c}\text { Esquerda } \\
\text { Média } \pm \text { Desvio } \\
\text { Padrão }\end{array}$ \\
\hline LONG & $100,12 \pm 4,01$ & $101,14 \pm 4,42$ & 0,3201 \\
TRANS & $13,50 \pm 1,72$ & $14,46 \pm 1,75$ & 0,8648 \\
FVENTRAL & $13,11 \pm 1,304$ & $14,01 \pm 1,317$ & 0,3722 \\
COND & $19,03 \pm 1,89$ & $17,02 \pm 2,071$ & 0,7130 \\
COR & $27,55 \pm 2,73$ & $28,12 \pm 2,06$ & 0,5412 \\
ANG & $21,94 \pm 2,531$ & $21,45 \pm 2,457$ & 0,4516 \\
\hline
\end{tabular}

Para a variável TRANS direita foi observado uma média de 13,50 mm com desvio padrão de $1,72 \mathrm{~mm}$, e para TRANS esquerda a média foi de 14,46 mm e desvio padrão de 1,75 mm. Na comparação entre os dois antímeros, o resultado de $p=0,8648$, não havendo portanto diferença significativa.

Analisando a variável FVENTRAL direita, a média encontrada foi de 13,11 $\mathrm{mm}$ e desvio padrão de 1,304 mm, e para FVENTRAL esquerda a média foi de $14,01 \mathrm{~mm}$ e desvio padrão de $1,317 \mathrm{~mm}$, o teste t-Student entre os dois antímeros revelou resultado de $p=0,3722$, não havendo diferença significativa.

Para a variável COND, as médias e desvios padrões foram de 19,03 \pm 1,89 $\mathrm{mm}$ para a hemimandíbula direita e 17,02 $\pm 2,071 \mathrm{~mm}$ para a hemimandíbula esquerda. O teste t-Student revelou resultado de 0,7130 não havendo diferença significativa entre os antímeros.

Também a variável COR não mostrou diferença significativa entre os antímeros com $p=0,5412$ e com médias e desvios padrões de 27,55 $\pm 2,73$ 
BARROSO, R.M.V. et al. Análise morfométrica do forame mandibular em Cachorro do Mato Vinagre (Speothos venaticus, LUND 1842). PUBVET, Londrina, V. 8, N. 14, Ed. 263, Art. 1744, Julho, 2014.

mm para a hemimandíbula direita e $28,12 \pm 2,06 \mathrm{~mm}$ para a hemimandíbula esquerda.

Considerando a variável ANG direita, a média foi de 21,94 mm com desvio padrão de 2,531 $\mathrm{mm}$ e para ANG esquerda, média de 21,45 $\mathrm{mm}$ e desvio padrão de 2,457. Na comparação entre os dois antímeros direito e esquerdo pelo teste t-Student, o resultado foi de 0,4516 também não indicando diferença significativa.

\section{DISCUSSÃO}

Estudos morfométricos ósseos tem sua aplicação no diagnóstico clínico em todas as espécies, em correlações como por exemplo, o risco de câncer nasal com o tipo de crânio, traçar variações na anatomia normal, e também, na determinação ósseo-arqueológica (REGODON et al., 1991; ONAR, 1999).

A presença de um forame mandibular na face medial do ramo da mandíbula, em posição ventral a inserção do músculo temporal e servindo como abertura caudal para o canal mandibular também foi verificado no Cachorro do Mato Vinagre (Speothos Venaticus) na mesma localização descrita para cães domésticos e Cachorro do Mato, corroborando com (SOUZA JÚNIOR, et al, 2013),

Em humanos, o bloqueio do nervo alveolar inferior, comumente (mas imprecisamente) denominado bloqueio nervoso mandibular, é a técnica de injeção mais usada e, possivelmente, a mais importante em odontologia. Infelizmente, também é a mais frustrante e a que possui maior porcentagem de fracassos clínicos (aproximadamente 15 a 20\%) (POTOCNIK \& BAJROVIC, 1999; MALAMED, 2001).

Diversos autores divergem sobre a exata localização do forame mandibular em pequenos animais, e somente Gross et al. (2000) especificou que o estudo foi realizado em gatos. Skarda (1996) e Fantoni \& Cortopassi 
BARROSO, R.M.V. et al. Análise morfométrica do forame mandibular em Cachorro do Mato Vinagre (Speothos venaticus, LUND 1842). PUBVET, Londrina, V. 8, N. 14, Ed. 263, Art. 1744, Julho, 2014.

(2002) indicam a posição a $5 \mathrm{~mm}$ rostral ao processo angular e de 10 a $20 \mathrm{~mm}$ dorsal a margem da mandíbula. Já Muir et al. (2001) afirma que o posicionamento se dá a $15 \mathrm{~mm}$ rostralmente ao processo angular e $15 \mathrm{~mm}$ dorsalmente a margem da mandíbula.

Assim como descrito por Getty (1986); Evans \& De Lahunta (1994); Boyd (1987) e König \& Liebich (2004) também foi encontrado neste estudo o ramo da mandíbula com sua superfície medial apresentando uma depressão rasa para inserção do músculo temporal, e ventral a esta, foi localizado o forame mandibular que é a abertura do canal mandibular.

Nicholson (1985), Blanton \& Roda (1995); Afsar et al. (1998) e Potocnik \& Bajrovic (1999), afirmam existir diferenças no posicionamento do forame mandibular em humanos, o mesmo ocorrendo no estudo com gatos domésticos (Barroso, et al, 2009), onde também foram encontradas diferenças significativas nos processos angulares, e coronóides entre os antímeros mandibulares.

A análise morfométrica do forame mandibular tanto em cães como em gatos, não é descrita na literatura anatômica, sendo encontrado dados somente na literatura referente à anestesia. Entretanto, Groos et al. (2000) descrevem esta análise em gatos, mas sem comparar os antímeros. Já Souza Júnior (2013), realizou estudo em Cachorro do Mato (Cerdocyon thous) também avaliando o posicionamento do forame mandibular, não encontrando variação entre antímeros e sugerindo a inserção de agulhas para procedimento anestésico local deve ser de 20,4 $\mathrm{mm}$ obliquamente a partir do processo angular e 12,1 mm (fêmeas) e 13,6 mm (machos) perpendicularmente a partir da borda ventral mandibular. Neste estudo foi observada diferença entre o sexo dos animais, fato que em nosso estudo não pode ser avaliado devido a peças já estarem coletadas e preparadas para osteologia, não permitindo a diferenciação por gênero.

Muir et al. (2001), afirma que para se obter sucesso no procedimento anestésico local do nervo alveolar inferior é necessário se introduzir a agulha a 
BARROSO, R.M.V. et al. Análise morfométrica do forame mandibular em Cachorro do Mato Vinagre (Speothos venaticus, LUND 1842). PUBVET, Londrina, V. 8, N. 14, Ed. 263, Art. 1744, Julho, 2014.

$15 \mathrm{~mm}$ rostralmente ao processo angular e $15 \mathrm{~mm}$ dorsalmente a margem ventral da mandíbula em gatos domésticos.

Já Skarda (1996) e Fantoni \& Cortopassi (2002), afirmam que é necessário se introduzir a agulha $5 \mathrm{~mm}$ rostralmente ao processo angular e entre 10 e $20 \mathrm{~mm}$ dorsalmente a margem da mandíbula.

Levando em consideração as duas variáveis adotadas pelos autores citados acima, encontramos como resultados a distância do forame mandibular até a margem ventral da mandíbula entre 13,11 e 14,01 mm sem diferença significativa entre os antímeros, e do processo angular até o forame mandibular de $21,94 \mathrm{~mm}$ para a mandíbula direita e $21,45 \mathrm{~mm}$ para a mandíbula esquerda, também sem a presença de diferenças significativas entre os antímeros. Nossos resultados se aproximaram dos obtidos por Paulo Júnior, et al (2013), sendo estes os únicos autores que realizaram análise do morfométrica do forame manbibular em canídeos selvagens. Estas medidas com aproximação eram esperadas tendo em vista o tamanho médio dos animais serem próximos.

\section{CONCLUSÃO}

Não foi observada uma variabilidade significativa no posicionamento do forame mandibular entre os antímeros de Cachorro do Mato Vinagre (Speothos Venaticus) em nenhuma das variáveis mensuradas.

Para um melhor bloqueio anestésico local do nervo alveolar inferior, a agulha deverá ser introduzida de 13,11 e 14,01 mm da margem ventral da mandíbula entre 21,94 a $21,45 \mathrm{~mm}$ partindo-se do processo angular.

\section{FINACIAMENTO}

O referido trabalho tem financiamento da Fundação de Amparo a Pesquisa do Espírito Santo - FAPES, através de bolsa de iniciação científica. 


\section{REFERÊNCIAS}

1. AFSAR, A.; HAAS, D. A.; ROSSOUW, E; WOOD, R. E. Radiographic localization of mandibular anesthesia landmarks. Oral Surgery, Oral Medicine, Oral Pathology, Oral Radiology Endodontics, Saint Louis, v. 86, p. 234-241, 1998.

2. BARROSO, R.M.V., FERREIRA, F. A., SILVA, R.M., LIMA, E.M.M., Morphometric analysis of the mandibular foramen of cats (Felis catus, LINNAEUS 1758) sem raça definida, Bioscience Journal, v. 25, n.4, p. 135-142, july/aug 2009

3. Beisiegel,B.M., Zuercher G. (2005) Speothos venaticus. Mammalian Species 783: 1-6. American Society of Mammalogists

4. BLANTON, P.L.; RODA, R.S. The anatomy of local anesthesia. California dental associaton journal, v. 23, p. 55-69, 1995.

5. BOYD, J. S. Anatomia clínica. 1 ed. São Paulo: Manole, 1997. 169 p.

6. DeMatteo K., Loiselle B. (2008) New data on the status and distribution of the bush dog (Speothos venaticus): Evaluating its quality of protection and directing research efforts. Biological Conservation 141: 2494-2505.

7. EVANS, H.E.; DE LAHUNTA, A. Guia para a dissecação do cão. 3 ed. Rio de Janeiro: Guanabara Koogan, 1994. 206 p.

8. FANTONI, D.T.; CORTOPASSI, S.R.G. Anestesia em cães e gatos. 1 ed. São Paulo: Roca, 2002. 389p.

9. GETTY, R. Sistema nervoso periférico. In: GETTY, R. Sisson/Grossman Anatomia dos animais domésticos. 5 ed. Rio de Janeiro: Guanabara Koogan, 1986, v. 2, p. 15831595.

10. GROSS, M.E.; POPE, E.R.; JARBOE, J.M.; O'BRIEN, D.P.; DODAM, J. R.; POLKOWHAIGHT, J. Regional anesthesia of the infraorbital and inferior alveolar nerves during noninvasive tooth pulp stimulation in halothane-anesthetized cats. American journal of veterinary research. Chicago, v. 61, n. 10, p. 1245-1247, 2000

11. Kleiman DG (1972) Social Behavior of the Maned Wolf (Chrysocyon brachyurus) and Bush Dog (Speothos venaticus): A Study in Contrast. Journal of Mammalogy 53: 791806.

12. KÖNIG, H. E.; LIEBICH, H. Anatomia dos animais domésticos, v. 2, ed. Rio de Janeiro: Artmed, 2004, 399 p.

13. MALAMED, S. F. Manual de anestesia local. 4 ed. Rio de Janeiro: Guanabara Koogan, 2001, 279 p.

14. MILKEN, V. M. F. Bloqueio do nervo alveolar mandibular com ropivacaína a 0,5\% em gatos sem raça definida. Dissertação de mestrado, Universidade Federal de Uberlândia, Programa de Pós-Graduação em Ciências Veterinária, Clínica e Cirurgia, 30 p., 2003.

15. MUIR III, W.W.; HUBBELL, J.A.E.; SKARDA, R.T.; BEDNARSKI, R.M. Manual de anestesia veterinária. 3 ed. São Paulo: Artmed, 2001. 432 p.

16. NICHOLSON, M.L. A study of the position of the mandibular foramen in the adult human mandible, The anatomical records, v. 212, p. 110-112, 1985.

17. NICKEL, R.; SCHUMMER, A.; SEIFERLE, E. The anatomy of the domestic animals. Berlim: Verlag Paul Parey, 1981, v.1, p 141. 
18. Oliveira TG (2009) Distribution, habitat utilization and conservation of the Vulnerable bush dog Speothos venaticus in northern Brazil. Oryx 43: 247-253.

19. Oliveira TG, Dalponte JC (2008) Speothos venaticus (Lund, 1842). In: Machado ABM, Drummond GM, Paglia AP (eds). Livro vermelho da fauna brasileira ameaçada de extinção. Volume II. Brasília, DF : MMA; Belo Horizonte, MG: Fundação Biodiversitas. pp. 783-784. Orr HA (2009) Fitness and its role in evolutionary genetics. Nat Rev Genet 10: 531-539.

20. ONAR, V. A Morphometric study on the skull of the german shepherd dog (Alsatian). Anatomy, Histology and Embriology, n. 28, p. 253-256, 1999.

21. POTOCNIK, I.; BAJROVIC, F. Failure of inferior alveolar nerve block in endodontics. Endodontics \& Dentral Traumatology, n. 15, p. 247-251, 1999.

22. REGODON, S.; ROBINA, A.; FRANCO, A.; VIVO, J. M.; LIGNEREUX, Y. Détermination radiologique et statistique dês types morphometriques crâniens chez lê chien: dolichocéphalie, mésocéphalie et brachycéphalie. Anatomy, Histology and Embriology, n. 20, p. 129-138, 1991.

23. SKARDA, R.T. Local and regional anesthesic and analgesia techniques: dogs. In: Lumb \& Jones veterinary anesthesia. 3. ed. Baltimore, Wiliams \& Wilkins, pp. 426-47, 1996.

24. Souza Junior, P.; Rodrigo Jiddu Ferreira Pinto, Adriano Biase Freitas, Natan Cruz Carvalho. Morfometria do forame mandibular de Cerdocyon thous (Linnaeus, 1766) (cachorro-do-mato) Biotemas, 26 (2): 175-183, junho de 2013

25. Zuercher G., Swarner M, Silveira L, Carrillo O (2008) Speothos venaticus. In: IUCN 2010. IUCN Red List of Threatened Species. Version 2010.1. <www.iucnredlist.org>. Downloaded on 07 de outubro de 2013.

Trabalho realizado através de bolsa de iniciação científica da Fundação de Amparo a Pesquisa do Espírito Santo - FAPES 